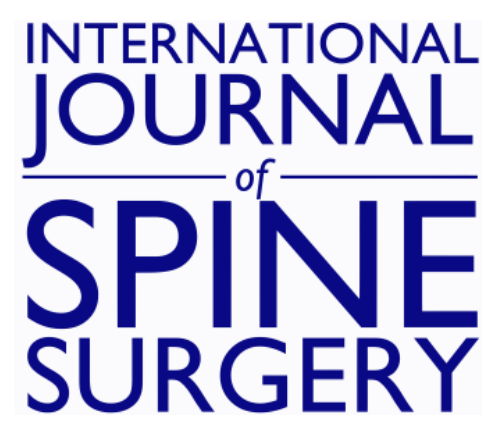

\title{
Postero-Lateral Disc Prosthesis Combined With a Unilateral Facet Replacement Device Maintains Quantity and Quality of Motion at a Single Lumbar Level
}

Aniruddh N. Nayak, Michael C. Doarn, Roger B. Gaskins III, Chris R. James, Andres F. Cabezas, Antonio E. Castellvi and Brandon G. Santoni

Int J Spine Surg 2014, 8 ()

doi: https://doi.org/10.14444/1031

http://ijssurgery.com/content/8/31

This information is current as of April 26, 2023.

Email Alerts Receive free email-alerts when new articles cite this article. Sign up at: http://ijssurgery.com/alerts 


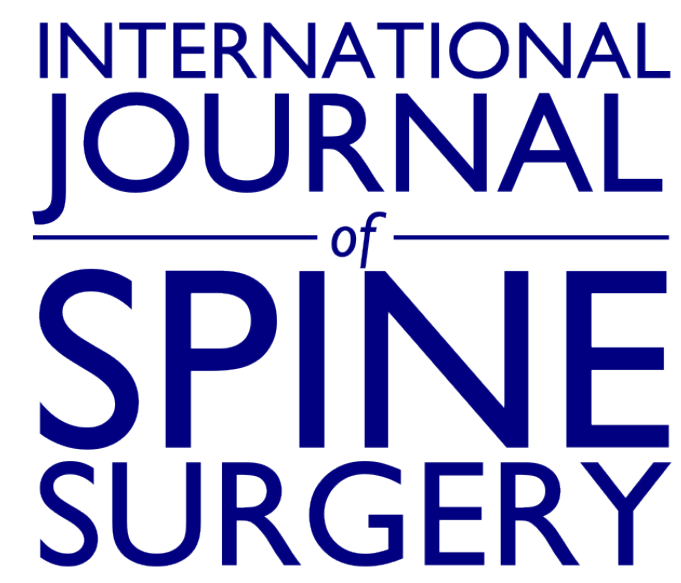

This article generously published free of charge by the International Society for the Advancement of Spine Surgery.

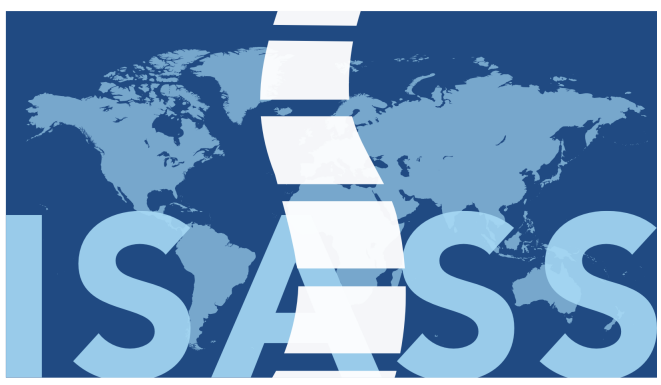

INTERNATIONAL SOCIETY for the ADVANCEMENT of SPINE SURGERY 


\section{Postero-Lateral Disc Prosthesis Combined With a Unilateral Facet Replacement Device Maintains Quantity and Quality of Motion at a Single Lumbar Level}

Aniruddh N. Nayak, MS, ${ }^{1}$ Michael C. Doarn, MD, ${ }^{2}$ Roger B. Gaskins, III, MD, ${ }^{2}$ Chris R. James, $M D,{ }^{3}$ Andres F. Cabezas, MS, ${ }^{1}$ Antonio E. Castellvi, MD, ${ }^{4}$ Brandon G. Santoni, Ph.D ${ }^{1,2}$

${ }^{1}$ Phillip Spiegel Orthopaedic Research Laboratory, Foundation for Orthopaedic Research \& Education, Tampa, FL, USA ${ }^{2}$ Department of Orthopaedics \& Sports Medicine, University of South Florida, Tampa, FL, USA ${ }^{3}$ Missouri Orthopaedic Institute, University of Missouri, Columbia, MO, USA ${ }^{4}$ Center for Spinal Disorders, Florida Orthopaedic Institute, Tampa, FL, USA

\section{Abstract}

\section{Background}

Mechanically replacing one or more pain generating articulations in the functional spinal unit (FSU) may be a motion preservation alternative to arthrodesis at the affected level. Baseline biomechanical data elucidating the quantity and quality of motion in such arthroplasty constructs is non-existent.

\section{Purpose}

The purpose of the study was to quantify the motion-preserving effect of a posterior total disc replacement (PDR) combined with a unilateral facet replacement (FR) system at a single lumbar level (L4-L5). We hypothesized that reinforcement of the FSU with unilateral FR to replace the resected, native facet joint following PDR implantation would restore quality and quantity of motion and additionally not change biomechanics at the adjacent levels.

\section{Study Design}

In-vitro study using human cadaveric lumbar spines.

\section{Methods}

Six $(\mathrm{n}=6)$ cadaveric lumbar spines (L1-S1) were evaluated using a pure-moment stability testing protocol $( \pm 7.5 \mathrm{Nm})$ in flexion-extension $(\mathrm{F} / \mathrm{E})$, lateral bending $(\mathrm{LB})$ and axial rotation (AR). Each specimen was tested in: (1) intact; (2) unilateral FR; and (3) unilateral FR + PDR conditions. Index and adjacent level ROM (using hybrid protocol) were determined opto-electronically. Interpedicular travel (IPT) and instantaneous center of 
rotation (ICR) at the index level were radiographically determined for each condition. ROM, ICR, and IPT measurements were compared (repeated measures ANOVA) between the three conditions.

\section{Results}

Compared to the intact spine, no significant changes in F/E, LB or AR ROM were identified as a result of unilateral FR or unilateral FR + PDR. No significant changes in adjacent L3-L4 or L5-S1 ROM were identified in any loading mode. No significant differences in IPT were identified between the three test conditions in F/E, LB or AR at the L4-L5 level. The ICRs qualitatively were similar for the intact and unilateral FR conditions and appeared to follow placement (along the anterior-posterior (AP) direction) of the PDR in the disc space

\section{Conclusion}

Biomechanically, quantity and quality of motion are maintained with combined unilateral FR + PDR at a single lumbar spinal level.

keywords:

total disc replacement, biomechanics, facet replacement, interpedicular travel, instantaneous center of rotation, lumbar spine arthroplasty

Volume 8 Article 31 doi: 10.14444/1031

\section{Introduction}

Non-traumatic low back pain (LBP) in adults is multifactorial and may result from degenerative disc disease (DDD), an impinging herniated disc, degenerated facet (mechanoreceptors) joints or a combination of these pathologies. ${ }^{1-3}$ The standard surgical approach to addressing pain in the degenerated functional spinal unit (FSU) in patients refractory to conservative treatment has been discectomy and fusion at the affected motion segment. Although fusion has been historically successful in relieving pain, a growing body of clinical and biomechanical data ${ }^{4-7}$ suggests that in the long term adjacent level degeneration may result, eventually requiring further surgical intervention. The objective of disc arthroplasty in the treatment of discogenic back pain is to replace the degenerated or herniated disc and restore sagittal balance while preserving normal motion with a mechanical surrogate device. ${ }^{8-10}$ By restoring motion, disc arthroplasty devices may circumvent the need for fusion and may prevent abnormal stresses at the adjacent levels. $^{11}$

From an anatomical perspective, the FSU is a tri-joint complex comprised of the disc and two facet joints. The disc confers six degrees of freedom to the FSU while facets guide flexion-extension motion, limit torsional motion and sustain $10-30 \%$ of axial and shear loads applied to the spine. ${ }^{12,13}$ The interplay and synergy between these structures during the degenerative stages is not clearly understood and the nature of the process differs between the disc and facet joints. However, due to their close relationship and function, degenerative changes or instability in one joint invariably affect the biomechanics of the remaining two. Prior work ${ }^{14}$ has demonstrated this paralleled degenerative relationship and facet arthrosis is a highly prevalent contraindication to total disc arthroplasty, 
occurring at an incidence of up to $97 \%$ in some clinical series. ${ }^{14,15}$ Furthermore, recent reports have demonstrated that the design and placement of disc arthroplasty devices may affect stresses and kinematics in the otherwise normal facet joint. ${ }^{16-21}$ These abnormal kinematic and kinetic changes may lead to the progression of facet arthrosis even when absent at the time of motion preserving surgery.

Clinical and biomechanical reports ${ }^{22-24}$ on outcomes associated with total facet replacements are sparse in the literature likely because facet replacement technology is relatively new compared to total disc replacements. Emerging total disc replacement designs utilize lateral trans-psoas and postero-lateral approaches to the degenerated disc. Postero-lateral approaches may be advantageous at limiting damage to vital vascular anterior-lateral structures; however, such an approach for disc replacement necessitates a unilateral facetectomy, which, in the biomechanical literature, has been shown to significantly increase ROM in some motion planes at the affected level, thereby resulting in instability at the FSU. One theoretical approach to restore posterior stability to the FSU after posterior disc replacement is to supplement the segment with a unilateral facet replacement device. Therefore, as a preliminary first step to test the efficacy of combined arthroplasty reconstruction of an FSU, we investigated range of motion (ROM), interpedicular travel (IPT), and centers of intervertebral rotation (ICR) at a single lumbar level following a reconstruction with a unilateral facet replacement in combination with a posterior-lateral total disc replacement.

\section{Materials and Methods}

\section{Specimen Preparation}

Six $(n=6)$ lumbar spines (L1-S1) were dissected from fresh-frozen, human cadaveric specimens ( 4 male/ 2 female; age range: 55 - 70 yrs.; mean age: $65 \pm 5$ years,). The medical history of each donor was reviewed to exclude specimens with trauma, malignancy, or significant metabolic disease. Radiographs were taken to confirm that specimens were free of significant deformity, prior instrumentation, bridging osteophytes and degenerated facet joints or discs. Specimens were cleaned and denuded of musculature and adipose tissue while retaining all ligamentous and bony structures. Bone mineral density (BMD) values (L1-L4) were assessed using dual energy x-ray absorptiometry (DEXA, Lunar Prodigy Advance, GE Healthcare, Waukesha, WI, USA) using an approach previously described for assessing bone quality in cadaveric tissue specimens denuded of extraneous soft tissues published by Wähnert et al. ${ }^{25}$ Average $\mathrm{BMD}$ and T-Scores were $1.0 \pm 0.2 \mathrm{~g} / \mathrm{cm}^{2}$ and $-1.6 \pm 1.6$, respectively.

Specimens were then rigidly potted at the cephalad and caudal (L1 \& S1) ends using interference screws and high strength fiberglass resin (Bondo, 3M, St. Paul, MN). Radiopaque lead beads (1.0 mm diameter) were implanted under fluoroscopy in the anterior and posterior aspect of the L3, L4, L5 and S1 endplates and unilaterally on the superior vertebral notches of the pedicles for each specimen. The beads served as fiducial markers for the radiographic assessment of the parameters such as intervertebral center of rotation (ICR) and inter-pedicular travel (IPT) that describe the qualitative aspect of motion. 
At the L4 and L5 levels, unilateral pedicle screws used as part of the facet replacement instrumentation (Figure 1) were implanted prior to attaching the follower load apparatus so that appropriate length screws could be used for affixing the compressive follower load system, thereby avoiding interference with pedicle screws. The follower load (FL) components and cable guides were attached mid-level to the L2, L3, L4 and L5 vertebrae using three fixation screws (two laterally and one anteriorly) and positioned approximately parallel to the endplates of the respective vertebral body and symmetrical to the mid-sagittal plane. In order to apply the FL, bilateral steel cables were passed caudally from the L1 superior vertebra potting via the cable guides attached to the FL apparatus towards the bilateral pulley guides on the S1 potting. A $400 \mathrm{~N}$ compressive load using dead weights was attached distally to it. The cable guides were adjusted in the anterior-posterior direction such that the path of the cables passed through the approximate location of the CORs of the motion segments and followed the lordotic curvature of the lumbar spine in accordance with the approach suggested by Patwardhan et al. (Figure 2). ${ }^{26}$ All cadaveric specimens were kept hydrated throughout dissection, instrumentation and biomechanical evaluation by wrapping with saline soaked gauze and spraying regularly with $0.9 \%$ saline. Prior to biomechanical testing, all specimens were thawed overnight (8-10 hours) at and tested at room temperature. 

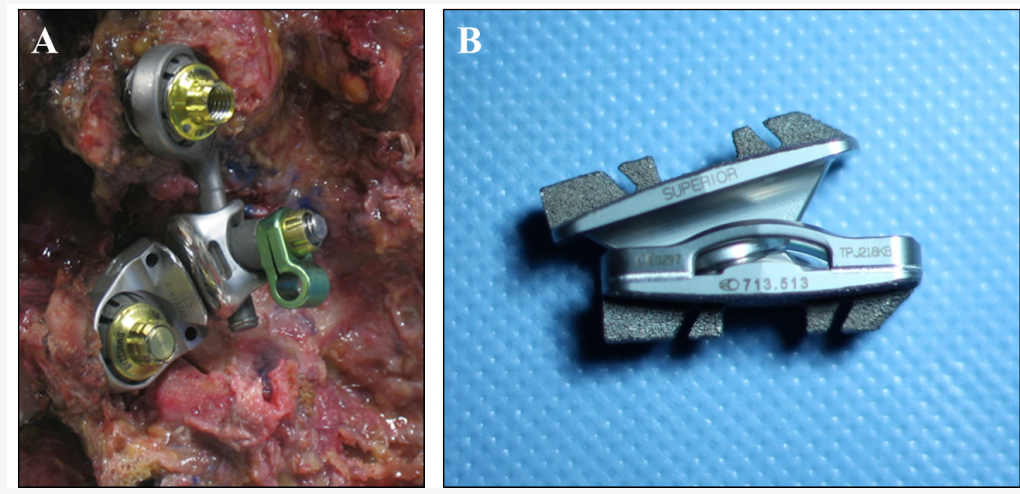

Fig. 1. Images of the facet (A) and posterior total disc (B) replacement devices.
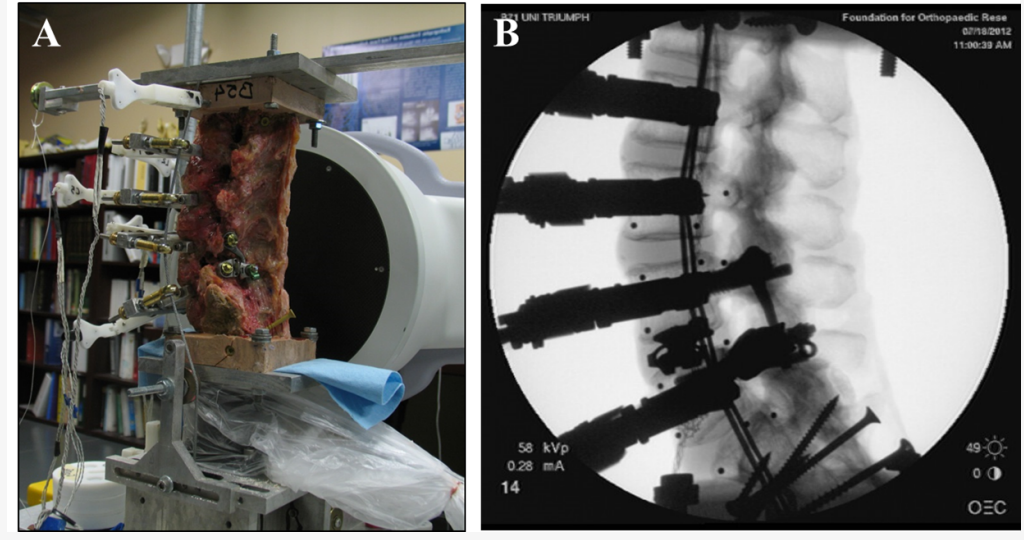

Fig. 2. Biomechanical testing set up showing moment application rod (A) attached at cranial potting and $400 \mathrm{~N}$ follower load (A, B) using cable guides, steel cables and dead weights (not shown in figure).

\section{Biomechanical Evaluation}

Biomechanical testing was accomplished using a pure-moment flexibility protocol using a system of cables and pulleys in similar fashion to previously reported methods. ${ }^{27-29}$ The caudal base (S1) of the specimen was mounted on a 6-component load cell (Model MC3A-1000, AMTI Transducers, Watertown, MA) and the specimen was allowed to move freely at the cephalad (L1) end. Pure moments up to $7.5 \mathrm{~N}-\mathrm{m}$ in increments of 1.5 $\mathrm{N}-\mathrm{m}$ were applied in flexion-extension (with and without a $400 \mathrm{~N}$ follower load), lateral bending (right + left), and axial rotation (right + left) for the intact and instrumented conditions. Fluoroscopic imaging (GE OEC FlexiView 8800, GE Medical, Waukesha, WI) was used during flexion and extension to monitor device motion and facilitate calculation of intervertebral center of rotation. To overcome the spine's viscoelastic effects, for each loading scenario, three preconditioning cycles up to $7.5 \mathrm{~N}-\mathrm{m}$ were applied to the specimen, and incrementally applied moments were maintained for approximately 30 seconds before recording range of motion (ROM). 
Optoelectronic rigid body marker triads (Optotrak Certus, Northern Digital Inc., Waterloo, Ontario, CA) were secured to each vertebral body. With the use of a digitizing probe, a local coordinate system for each vertebra was defined using three anatomical landmarks per body using the convention recommended by Panjabi and White. ${ }^{30}$ Unilaterally placed pedicle beads were also digitized to facilitate three dimensional (3D) IPT measurements. Relative intervertebral ROM was calculated as the Euler/cardan angles corresponding to rotations about the three axes of motion (Flex/Ext, R/L bending and $\mathrm{R} / \mathrm{L}$ axial rotation). Changes in adjacent level motion as a function of instrumentation were derived according to the Hybrid Testing Protocol proposed by Panjabi ${ }^{31}$ in which the total angular displacement recorded for the intact moment-controlled condition served as the displacement input for subsequent loading conditions of the instrumented spine.

Changes in the relative position of the pedicle at L4-L5 index level between peak planar loading conditions $( \pm 7.5 \mathrm{Nm})$ was described using an IPT kinematic metric previously introduced by Cook et al. ${ }^{32}$ The magnitude of the three dimensional vector was calculated by tracking the digitized bead that was placed unilaterally on the superior notch of the L4 and L5 pedicles. From Figure 3, the vector $r_{3}$ (dashed) and can be expressed as its components $\left(r_{x}, r_{y}\right.$, and $\left.r_{z}\right)$ in each of the reference coordinate axes $(x, y, z)$. The magnitude of this vector is defined as:

IPT $=\left\|r_{3}\right\|=\sqrt{ }\left(r_{\mathbf{x}}^{2}+r_{\mathrm{y}}^{2}+r_{z}^{2}\right)$ 


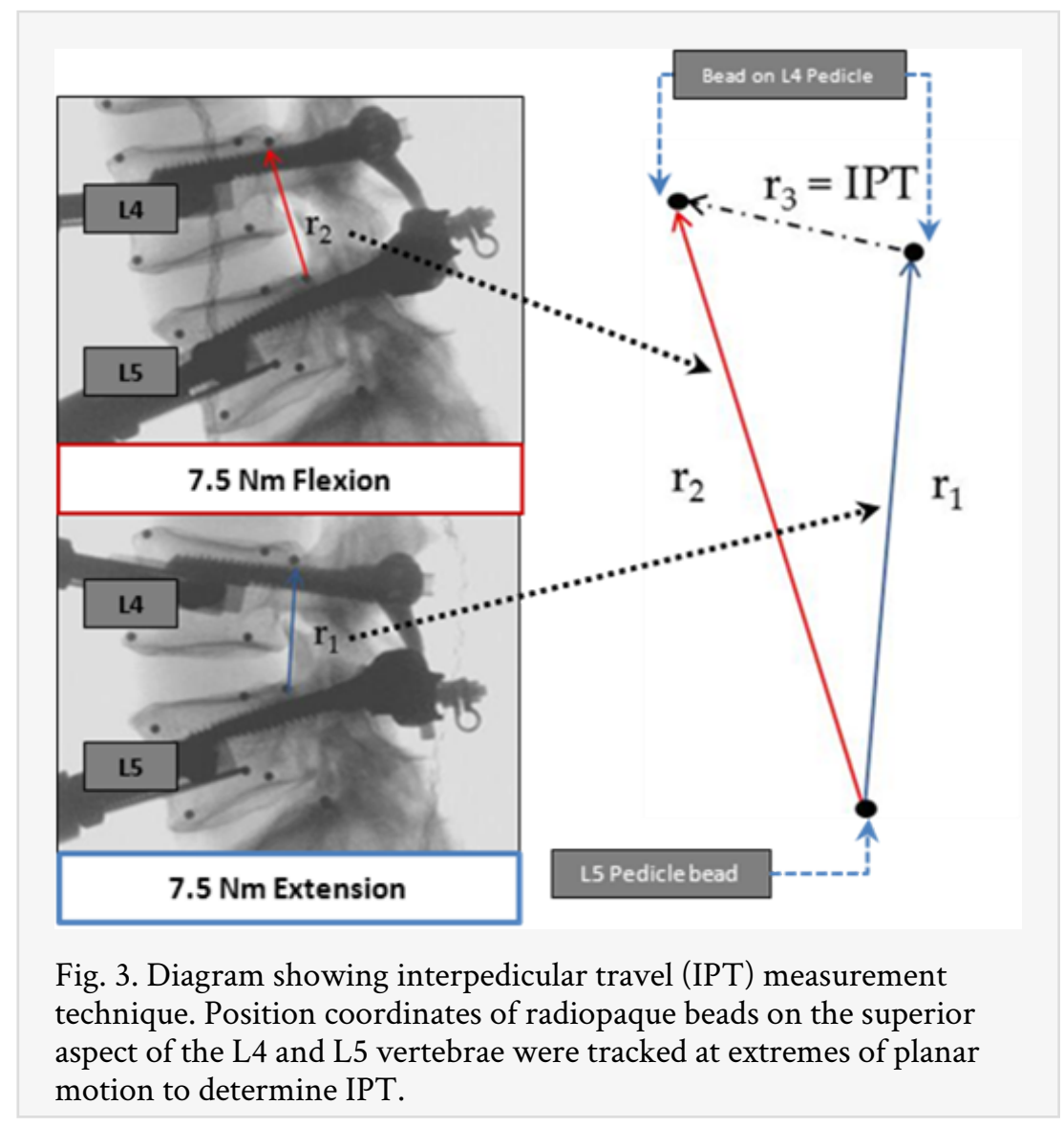

IPT is defined as the scalar of the linear distance through which the pedicles moves relative to one another over one FSU during flexion/extension, lateral bending and axial rotation motion as a function of treatment condition. The 3D coordinates of the embedded lead shot were determined by digitizing them prior to kinematic evaluation. Positional data at the extremes of pure moment loading $(+/-7.5 \mathrm{~N}-\mathrm{m})$ in each loading mode were used to calculate IPT. To determine changes in sagittal plane ICR at the L4-L5 index level as a function of spinal reconstruction condition, lateral fluoroscopic images were obtained in the full flexion $(+7.5 \mathrm{~N}-\mathrm{m})$, Neutral $(0 \mathrm{Nm})$ and full extension $(-7.5 \mathrm{~N}-\mathrm{m})$ positions. Images were calibrated prior to manually obtaining $2 \mathrm{D}$ coordinate data of the four vertebral beads ( 2 on L4 inferior endplate and 2 on L5 superior endplate) using ImageJ (NIH, Bethesda, MD) for all three positions. This coordinate data were entered into a previously validated custom VB code (Microsoft Office, Visual Basic Suite, 2010, Seattle, WA) that calculated resultant coordinates of the index level COR and this location was mapped on the calibrated "Neutral" image using ImageJ.

\section{Specimen Reconstruction}

Following kinematic evaluation of the intact L1-S1 specimen, a complete unilateral facetectomy at the L4-L5 index level was performed. The disc was left intact. The resected facet was subsequently reconstructed (Figure 4A) and tested (Unilateral -FR) with an appropriately sized arthroplasty device (ACADIA Facet Replacement System, Globus Medical Inc., Audubon, PA). The FR has been designed to closely mimic natural anatomy, kinematics and range of motion of the joint and is made of cobalt chrome alloy 
(Co-Cr-Mo) with highly polished articulating surfaces. The backing of the implant that interfaces with bone is plasma sprayed and coated with hydroxyapatite to promote bony in-growth. The implant was secured to the pedicle screws that were previously instrumented with locking nuts. Right or left side resection and replacement of the FR in the unilateral condition was randomized such that there were 3 right and 3 left unilateral facet replacements to account for instrumentation bias. For the third condition, the unilateral FR was temporarily removed, and a single posterior-lateral total disc prosthesis (PDR) was implanted (Triumph Lumbar Disc, Globus Medical Inc.) postero-laterally in the disc space. The PDR is a semi-constrained device made of Co-Cr-Mo alloy and has serrated endplate keels coated with titanium plasma and hydroxyapatite for enhanced bony in-growth (Figure 1B, Figure 4B). The device allows up to $\pm 15^{\circ}$ of flexion/ extension and lateral bending motion, and restricts axial rotation to $\pm 3^{\circ}$ with a locking mechanism.

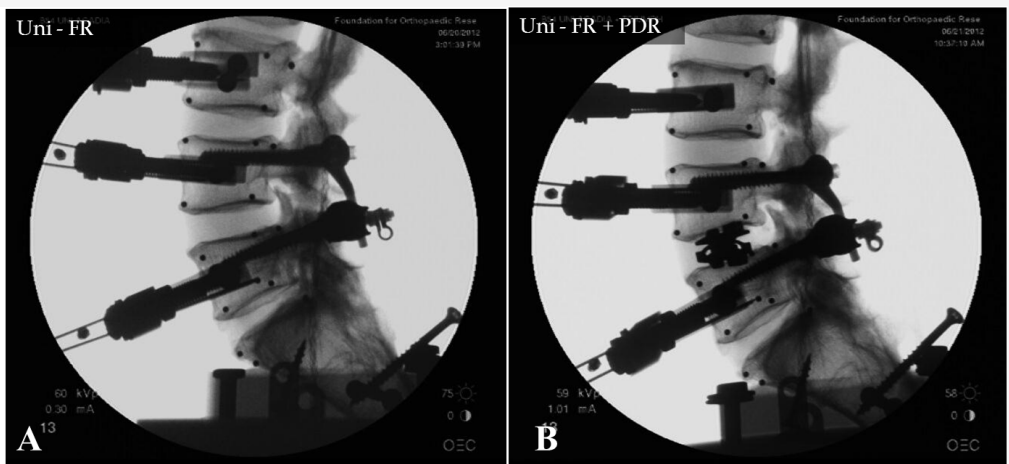

Fig. 4. Lateral radiographs indicating sequential index level (L4-L5) instrumentation with the facet and disc replacement devices. As shown [L-R]: Unilateral FR and Unilateral FR + PDR.

The superior and inferior components of the FR device were re-attached to the anchoring pedicle screws and the construct was tested in the unilateral FR with PDR condition (Uni FR + PDR). Implant sizing and instrumentation were performed according to manufacturer's recommended technique. A fellowship-trained spine surgeon performed all instrumentation procedures and all surgical interventions were performed and confirmed using fluoroscopy.

\section{Statistical Analysis}

Within each specimen, ROM changes between Intact, Uni-FR, and Uni-FR + PDR constructs were compared using a one-way repeated measures ANOVA and post hoc multiple comparison procedure with Bonferroni correction. Adjacent level motion and IPT measurements as a function of instrumented condition were compared using the same statistical approach. P-values less than 0.05 were considered significant. All statistical analyses were performed with SPSS.20 statistical software (IBM). 


\section{Results}

\section{Range of Motion \& IPT at the Operative L4-L5 Segment}

\section{Flexion-Extension}

Baseline total flexion-extension ROM for the intact L4-L5 segment was $11.3 \pm 4.3^{\circ}$

(Figure 5). Subsequent spinal reconstruction with the Uni-FR $\left(12.3^{\circ} \pm 4.3^{\circ}\right)$ and Uni-FR + $\operatorname{PDR}\left(10.3^{\circ} \pm 3.1^{\circ}\right)$ did not significantly affect flexion-extension ROM $(\mathrm{p}=0.081)$. Range of motion findings were similar under $400 \mathrm{~N}$ applied compressive follower load, with no significant differences in flexion-extension between instrumentation conditions $(\mathrm{p}=$ 0.747). Baseline flexion-extension IPT was $13.0 \pm 3.9 \mathrm{~mm}$ without the follower load and $10.2 \pm 3.5 \mathrm{~mm}$ with the follower load (Table 1). Without the follower load, IPT in the Uni-FR and Uni-FR+PDR conditions increased to $14.6 \pm 4.1 \mathrm{~mm}(\mathrm{p}=0.008)$ and $14.3 \pm$ $4.1 \mathrm{~mm}(\mathrm{p}=0.043)$. No changes in IPT during flexion-extension loading were noted with the follower load for either reconstruction condition $(\mathrm{p}=0.294)$.

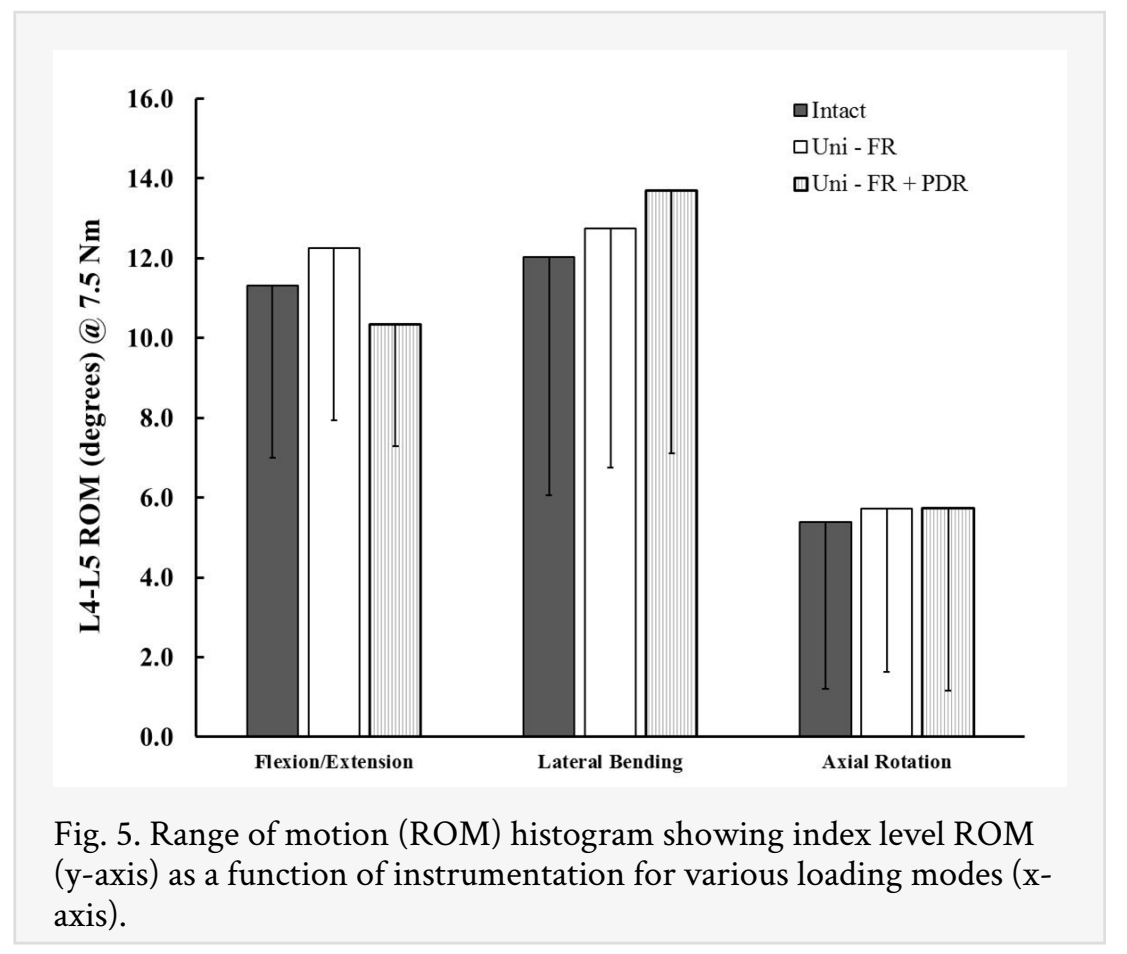

Table 1. Interpedicular Travel (IPT,mm) at index L4-5 level. Reported as Mean \pm Standard deviation.

\begin{tabular}{|l|l|l|l|}
\hline Loading Mode & Intact & Uni-FR & Uni-FR + PDR \\
\hline Flexion-Extension (No FL) & $130 \pm 3.9$ & $14.6 \pm 4.1^{1}$ & $14.3 \pm 3.8^{2}$ \\
\hline Flexion-Extension (FL)** & $10.2 \pm 3.5$ & $10.5 \pm 3.7$ & $11.5 \pm 3.0$ \\
\hline Lateral Bending** & $9.6 \pm 3.3$ & $10.7 \pm 3.8$ & $12.2 \pm 4.4$ \\
\hline Axial Rotation** & $3.5 \pm 1.8$ & $3.9 \pm 3.3$ & $4.8 \pm 5.3$ \\
\hline
\end{tabular}


${ }^{1}$ indicates Uni-FR IPT $>$ Intact IPT $(\mathrm{p}=0.008) ;{ }^{2}$ indicates Uni-FR+PDR IPT $>$ Intact IPT $(\mathrm{p}=0.043)^{* *}$ Indicates no significant difference $(\mathrm{p}>0.05)$ between instrumentation conditions

\section{Lateral Bending}

Intact total lateral bending ROM for the L4-L5 level was $12.0 \pm 6.0^{\circ}$ (Figure 5). No changes in bending ROM were noted for the Uni-FR $\left(12.7 \pm 6.0^{\circ}\right)$ or Uni-FR + PDR conditions $\left(13.7 \pm 6.0^{\circ}\right)(\mathrm{p}=0.185)$. Baseline total lateral bending IPT for the L4-L5 level was $9.6 \pm 3.3 \mathrm{~mm}$ (Table 1). No significant changes in lateral bending IPT were identified between the intact spine and the Uni-FR and Uni-FR + PDR conditions $(p=0.123)$.

\section{Axial Rotation}

Baseline total axial rotation ROM for the L4-L5 level was $5.4 \pm 4.2^{\circ}$ (Figure 5). Axial rotation ROM was not affected be Uni-FR $\left(5.7 \pm 4.2^{\circ}\right)$ or Uni-FR + PDR reconstruction $\left(5.8 \pm 4.6^{\circ}\right)$ relative to the intact level $(\mathrm{p}=0.478)$. Baseline total axial rotation IPT for the L4-L5 level was $3.5 \pm 1.8 \mathrm{~mm}$ (Table 1). Axial rotation IPT was not affected by progressive reconstruction with Uni-FR or Uni-FR + PDR $(\mathrm{p}=0.178)$.

\section{Motion at the Non-Operated Segments}

Baseline flexion-extension motions at the superior L3-L4 level with and without the 400 $\mathrm{N}$ follower load were $8.8 \pm 3.7^{\circ}$ and $8.8 \pm 4.1^{\circ}$, respectively (Table 2 ). No significant changes in L3-L4 flexion-extension ROM with or without the follower load were identified between the intact condition and progressive spinal arthroplasty $(p=0.312)$ constructs. Baseline flexion-extension motions at the inferior L5-S1 level were $11.7 \pm$ $4.2^{\circ}$ and $8.1 \pm 3.0^{\circ}$ with and without the $400 \mathrm{~N}$ follower load, respectively. No significant changes in ROM in this loading mode were identified between the intact L5-S1 level and the reconstruction conditions $(\mathrm{p}=0.215)$.

Table 2. Adjacent level ROM derived from Hybrid Loading Protocol [mean \pm stdev].

\begin{tabular}{|l|l|l|l|l|l|l|l|l|l|}
\hline & \multicolumn{7}{|l|}{ Instrumentation Condition at Index L4-L5 Level } \\
\hline Loading Mode & \multicolumn{2}{|l|}{ Intact } & \multicolumn{2}{l|}{ Uni-FR } & \multicolumn{3}{l|}{ Uni-FR + PDR } & \\
\hline Superior L3-L4 Level ** & & & & & & & \\
\hline Flexion-Extension ROM [w/o FL] & 8.8 & \pm & 3.7 & 8.5 & \pm & 3.7 & 8.9 & \pm & 4.2 \\
\hline Flexion-Extension ROM [w/400 N FL] & 8.8 & \pm & 4.1 & 8.6 & \pm & 4.2 & 9.3 & \pm & 5.2 \\
\hline Lateral Bending ROM & 11.4 & \pm & 5.9 & 12.1 & \pm & 4.7 & 11.7 & \pm & 6.7 \\
\hline Axial Rotation ROM & 5.5 & \pm & 2.6 & 5.4 & \pm & 2.1 & 5.6 & \pm & 2.3 \\
\hline Inferior L5-S1 Level ** & & & & & & & & \\
\hline Flexion ROM & 6.5 & \pm & 3.1 & 6.4 & \pm & 3.1 & 6.9 & \pm & 3.0 \\
\hline Flexion-Extension ROM & 8.1 & \pm & 3.0 & 8.5 & \pm & 3.9 & 8.9 & \pm & 4.3 \\
\hline Lateral Bending ROM & 8.0 & \pm & 2.9 & 8.1 & \pm & 2.9 & 8.8 & \pm & 3.3 \\
\hline Axial Rotation ROM & 4.1 & \pm & 1.2 & 4.1 & \pm & 1.2 & 4.6 & \pm & 1.5 \\
\hline
\end{tabular}

** Indicates no significant difference in adjacent level ROM for any loading mode $(p>0.05)$

Lateral bending ROM at the superior L3-L4 and inferior L5-S1 levels were not affected by progressive spinal arthroplasty at the L4-L5 level ( $p>0.123$ ). Axial rotation ROM at the adjacent levels similarly were not affected by arthroplasty reconstruction at L4-L5 (p $=0.178$ ). 


\section{Quality of Motion at the Operative L4-L5 Segment}

The calculated ICR at the instrumented level was plotted on lateral radiographs of a single specimen, not tested as part of the current experiment, in the intact and progressively instrumented conditions. A best-fit plot was created to demonstrate the relative changes in position of the centroid of motion as a function of instrumented condition (Figure 6). Overall, intact ICRs were generally localized in the anterior and middle columns of the L4-L5 FSU within the disc space and inferior to the superior endplate of L5. Unilateral facet arthroplasty after facetectomy (Uni-FR) qualitatively maintained the ICR in a location consistent with that of the intact FSU. The addition of the posterior total disc (Uni FR + PDR) resulted in a more diffuse pattern of ICR localized inferior to the superior L5 endplate. The ICRs appeared to qualitatively shift along the AP direction as a result of sagittal plane placement of the PDR within the disc space. A slightly posterior placement of the PDR within the disc space appeared to restore the ICR to near that of the intact FSU, while a more central placement shifted the ICR slightly in the anterior direction.
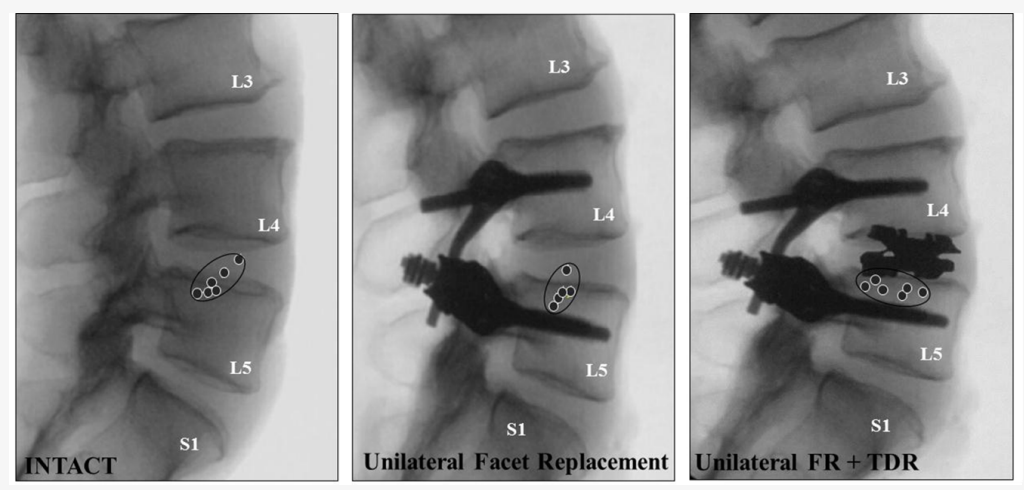

Fig. 6. Representative lateral radiographs with plot of the center of rotation for all six specimens at the L4-L5 level as a function of instrumentation.

\section{Discussion}

Total disc arthroplasty is gaining in popularity as a surgical alternative to fusion for the treatment of symptomatic back pain and degenerative disc disease. The posterior approach to disc replacement is biomechanically advantageous, as important stabilizers of the anterior spinal column are preserved, namely the anterior longitudinal ligament and anterior-lateral annulus. Clinically, surgeons are more familiar with posterior and posterolateral approaches and surgery does not require a vascular surgeon to assist with access to the disc space. The procedure thus is safer as the anterior vascular structures are not exposed. Implantation does, however, necessitate a unilateral resection of the facet joint and/or part of the lamina. As the facet joints are important stabilizers of the posterior spinal column, ${ }^{12,13}$ their removal has been demonstrated to biomechanically alter the normal kinematic trajectory of the FSU, affecting quantity of quality of motion and resulting in a loss of inherent stability. ${ }^{22}$ Combined with the prevalence of facet arthrosis as a common contraindication to disc arthroplasty ${ }^{14}$, emerging clinical ${ }^{33}$ and biomechanical ${ }^{34}$ data suggest that facet joint loading patterns are adversely affected by 
disc implantation which may ultimately be linked with an accelerated progression of facet arthrosis. The addition of a facet arthroplasty system unilaterally to re-establish posterior load sharing as well as the quantity and quality of the natural ROM may be a feasible treatment option when arthroplasty is indicated for surgical intervention. Prior to clinical use, biomechanical data are necessary to demonstrate the proof of concept and report the associated kinematics and quality (or pattern) of motion associated with such a non-fusion surgical treatment modality. Our study serves as the first comprehensive laboratory evaluation of these devices when incorporated simultaneously into a single lumbar level arthroplasty construct.

Definition of the 'quantity' of spinal motion has historically been accomplished with the use of pure moment or displacement loading protocols in combination with motion analysis systems that monitor, in real-time, intervertebral rotations during loading about the flexion-extension, bending and rotational axes of the FSU. Reporting the changes in ROM as a function of progressive instrumentation in cadaveric specimens is commonplace and important for comparative studies that evaluate new fusion and motion-sparing devices. However, reports of ROM only account for the dominant rotational motion, and translational effects that may be imparted to the FSU by the device are not taken into consideration when ROM alone is reported.

Interpedicular travel (or IPT) was recently introduced by Cook et al. ${ }^{32}$ as a kinematic parameter to assess quality of intervertebral motion. Since IPT is measured away from the center of rotation of the FSU, quantitatively it can be considered as a summation of both the rotational and translational motion of the FSU. A quantitative description of IPT may be of value in describing the effects of motion-preserving implants on spinal motion, particularly so when anterior and posterior column motion preservation prostheses are coupled together. As such, in the current study, we report both intervertebral ROM and IPT as metrics to determine whether the arthroplasty devices maintain the natural limits of motion in a single lumbar level. Our findings indicate the ROM was maintained at the index level relative to the intact condition with progressive arthroplasty at L4-L5, and are in general agreement with published biomechanical studies evaluating motion-preserving disc arthroplasty devices in cadaveric lumbar spines. ${ }^{35,36}$ Kikkawa et al. ${ }^{37}$ evaluated the same posterior total disc replacement investigated in this study. Following unilateral facetectomy and posterior total disc replacement, axial rotation ROM was increased by approximately $30 \%$ relative to the intact motion segment. In the current study, axial rotation ROM was maintained in the Uni FR + PDR constructs, suggesting that the facet replacement device can restore the kinematic stability lost after facetectomy and disc arthroplasty. The re-creation of the intimate contact between the articulating surfaces of the facet joint conferred by the facet arthroplasty device appears to be critical for controlling rotational motion. Measurements of IPT for all instrumented conditions in the same loading planes trended similarly as our ROM findings.

Total disc arthroplasty designs have traditionally incorporated various types of ball and socket mechanisms that are constrained, unconstrained or semi-constrained. These various design features guide the motion (translation and rotation) and dictate the COR of the reconstructed spinal level. Because of its unconstrained design, the FR allows motion secondary to the PDR, which imparts primary motion to the FSU. This finding was corroborated by the COR plot for the intact and instrumented conditions. The sagittal 
plane CORs in the Uni-FR + PDR condition were consistently in the posterior half of the disc space and located below the superior L5 endplate, which were approximately the geometric centers of the convex inferior components of the PDR device. Current study results with the PDR device tested here are in good agreement with those previously reported by Kikkawa et al., ${ }^{37}$ who reported that COR location was dictated by PDR placement within the disc space. In their study, the device was either placed in the anterior, middle, or posterior third of the disc space. Anterior placement of the device shifted the ICR anteriorly from the intact COR location, while central or posterior placement appeared to restore the COR to the intact condition. Our findings that the CORs of the Uni-FR + PDR construct were in the same approximate location reported by Kikkawa et al. using the PDR alone add additional support that the motion patterns conferred to the FSU were not competing or mismatched and that combining the FR with the PDR maintains the COR in a location similar to the intact L4-L5 spinal level. A better understanding of the combined synergistic biomechanical properties of existing disc and facet replacement devices in a controlled in vitro setup is required to carefully assess their clinical utility.

Equally important to understanding the effects of motion-preservation at the index level is the effect that the device imparts on adjacent segments. As reported here, ROM at the superior L3-L4 and inferior L5-S1 was maintained following progressive arthroplasty at the L4-L5 level. Our results regarding maintenance of adjacent level motion are in good agreement with previous biomechanical work evaluating various designs of disc arthroplasty prostheses used in the lumbar spine when tested under pure-moment loading conditions. ${ }^{36,38}$ Further, prior work by Phillips et al. ${ }^{22}$ has demonstrated that both index level and adjacent level kinematic signatures are maintained at near intact levels following facet arthroplasty with the predecessor device (TFAS, Archus Orthopaedics, Redmond, WA) to that used in the current study. Other published works support total facet arthroplasty as a viable biomechanical treatment for restoration of natural spinal kinematics. ${ }^{24,39}$ The most notable difference between these prior works and the current investigation is that we use the FR device only in a unilateral condition to answer our primary study question. However, future studies may evaluate the posterolateral disc in combination with bilateral facet replacement to determine if arthroplasty of the entire three-joint complex of the FSU maintains intact segment kinematics.

Here, the specimens were initially evaluated in a load-controlled (pure moment loading) test mode after each successive surgical procedure. Such an approach simulates a scenario in which the applied moments are identical pre- and post-operatively. To monitor the effects of hardware implantation at one level on the kinematics of adjacent levels, Panjabi and Goel $^{40}$ proposed a hybrid test protocol in which the pure moments are applied until the spine is achieves identical global (L1-S1) motion end limits before and after instrumentation. As would be the case with a simulated fusion, adjacent level motion would necessarily increase to reach the pre-surgical global ROM when this laboratory method is employed, and this has been reported previously by a number of authors. ${ }^{41,42}$ As demonstrated in the current study, when no significant changes in ROM at an index level are noted with the use of a motion-preserving device, then the kinematic signatures of the adjacent levels are at near-normal levels. While the clinical applicability of the 
hybrid test method is unclear, it does serve as a valuable laboratory tool to capture subtle changes in adjacent segment kinematics following an arthroplasty or arthrodesis procedure.

In the current study, we employed the follower load advocated by Patwardhan et al. ${ }^{26}$ to simulate the stabilizing compressive effects that the paraspinous musculature imparts to the spine in vivo. In its currently implemented form, the follower load can only be applied while assessing the kinematic response of the spine under flexion-extension loading. Due to its inherent technical limitations, consistent with every other study utilizing the follower load technique, it was not applied during lateral bending or axial rotation loading. Because of the stabilizing effect that the compressive pre-load applies to the spinal segments, the current ROM results pertaining to lateral bending and axial rotation can be considered a worst-case kinematic outcome. In vivo, one might expect lower ROM values in these loading modes due to the stability that the paraspinous musculature confers to the reconstructed segment.

Our study is not without limitations. Firstly, the nature of our biomechanical test method is only able to describe the acute kinematic effects of the unilateral facet replacement system and posterior total disc prosthesis. They cannot account for the repetitive nature of complex mechanical loading that will likely occur in the post-operative course and any progressive changes in the quantity and quality of FSU motion over that duration. A finite element modeling study for such a construct could shed more light on the load distribution/sharing properties and strains on the contralateral intact facet capsules and ligaments. The results reported herein also do not take into account the biological changes that occur in vivo and should not be extrapolated to time periods beyond the immediate post-operative. Secondly, though we attempted to control for the stabilizing, compressive effects of the paraspinous musculature with the follower load apparatus, we were only able to do so in one loading mode due to technical limitations associated with the pre-load technique. As such it is unknown how our biomechanical findings translate into the clinical setting in the treatment of patients with such an arthroplasty procedure. Certainly, additional biomechanical studies and meticulous classification of inclusion criteria are required prior to clinical implementation and determination of safe advancement of this novel concept.

In summary, the results of this study suggest that following unilateral facetectomy and posterior total disc replacement, use of a unilateral facet replacement is adequate enough to maintain both the quantity and pattern of FSU motion at the operated level. Future biomechanical work may investigate the effect of total disc replacement ini combination with bilateral facet replacement to determine if prosthetic replacement of the entire threejoint complex (i.e. $360^{\circ}$ arthroplasty) can adequately restore the quantity and quality of motion at a single affected lumbar level.

\section{Acknowledgements}

The authors acknowledge research and institutional support provided by Globus Medical Inc., Audubon, PA. 


\section{References}

1. Palepu V, Kodigudla M, Goel VK. Biomechanics of disc degeneration. Adv Orthop. 2012;2012:726210. PubMed PMID: 22745914. Pubmed Central PMCID: 3382964. Epub 2012/06/30. eng.

2. Kirkaldy-Willis WH, Hill RJ. A more precise diagnosis for low-back pain. Spine. 1979 Mar-Apr;4(2):102-9. PubMed PMID: 162546.

3. Kelsey JL, White AA, 3rd. Epidemiology and impact of low-back pain. Spine. 1980 Mar-Apr;5(2):133-42. PubMed PMID: 6446158.

4. Tu TH, Ziewacz JE, Mummaneni PV. Editorial: Disc replacement versus fusion. Journal of neurosurgery Spine. 2012 Dec;17(6):502; author response 3. PubMed PMID: 23082847.

5. Zigler JE, Glenn J, Delamarter RB. Five-year adjacent-level degenerative changes in patients with single-level disease treated using lumbar total disc replacement with ProDisc-L versus circumferential fusion. Journal of neurosurgery Spine. 2012 Dec;17(6):504-11. PubMed PMID: 23082849.

6. Imagama S, Kawakami N, Kanemura T, Matsubara Y, Tsuji T, Ohara T, et al. Radiographic Adjacent Segment Degeneration at Five Years After L4/5 Posterior Lumbar Interbody Fusion With Pedicle Screw Instrumentation: Evaluation by Computed Tomography and Annual Screening With Magnetic Resonance Imaging. J Spinal Disord Tech. 2013 (In Press) Feb 19. PubMed PMID: 23429323.

7. Helgeson MD, Bevevino AJ, Hilibrand AS. Update on the evidence for adjacent segment degeneration and disease. Spine J. 2013 Feb 15. PubMed PMID: 23420004.

8. Cunningham BW. Basic scientific considerations in total disc arthroplasty. Spine J. 2004 Nov-Dec;4(6 Suppl):219S-30S. PubMed PMID: 15541670. Epub 2004/11/16. eng.

9. Errico TJ. Lumbar disc arthroplasty. Clin Orthop Relat Res. 2005 Jun(435):106-17. PubMed PMID: 15930927. Epub 2005/06/03. eng.

10. Fekete TF, Porchet F. Overview of disc arthroplasty-past, present and future. Acta Neurochir (Wien). 2010 Mar;152(3):393-404. PubMed PMID: 19844656. Epub 2009/ 10/22. eng.

11. Dooris AP, Goel VK, Grosland NM, Gilbertson LG, Wilder DG. Load-sharing between anterior and posterior elements in a lumbar motion segment implanted with an artificial disc. Spine. 2001 Mar 15;26(6):E122-9. PubMed PMID: 11246394.

12. Serhan HA, Varnavas G, Dooris AP, Patwadhan A, Tzermiadianos M. Biomechanics of the posterior lumbar articulating elements. Neurosurg Focus. 2007;22(1):E1. PubMed PMID: 17608330. Epub 2007/07/05. eng.

13. Varlotta GP, Lefkowitz TR, Schweitzer M, Errico TJ, Spivak J, Bendo JA, et al. The lumbar facet joint: a review of current knowledge: part 1: anatomy, biomechanics, and grading. Skeletal Radiol. 2011 Jan;40(1):13-23. PubMed PMID: 20625896. Epub 2010/07/14. eng.

14. Wong DA, Annesser B, Birney T, Lamond R, Kumar A, Johnson S, et al. Incidence of contraindications to total disc arthroplasty: a retrospective review of 100 consecutive fusion patients with a specific analysis of facet arthrosis. Spine J. 2007 Jan-Feb;7(1):5-11. PubMed PMID: 17197326.

15. Huang RC, Lim MR, Girardi FP, Cammisa FP, Jr. The prevalence of contraindications to total disc replacement in a cohort of lumbar surgical patients. 
Spine. 2004 Nov 15;29(22):2538-41. PubMed PMID: 15543070. Epub 2004/11/16.

eng.

16. Rousseau MA, Bradford DS, Bertagnoli R, Hu SS, Lotz JC. Disc arthroplasty design influences intervertebral kinematics and facet forces. Spine J. 2006 MayJun;6(3):258-66. PubMed PMID: 16651219. Epub 2006/05/03. eng.

17. Schmidt H, Midderhoff S, Adkins K, Wilke HJ. The effect of different design concepts in lumbar total disc arthroplasty on the range of motion, facet joint forces and instantaneous center of rotation of a L4-5 segment. Eur Spine J. 2009 Nov;18(11):1695-705. PubMed PMID: 19763638. Pubmed Central PMCID: 2899406. Epub 2009/09/19. eng.

18. Botolin S, Puttlitz C, Baldini T, Petrella A, Burger E, Abjornson C, et al. Facet joint biomechanics at the treated and adjacent levels after total disc replacement. Spine. 2011 Jan 1;36(1):E27-32. PubMed PMID: 20975623. Epub 2010/10/27. eng.

19. Kafchitsas K, Kokkinakis M, Habermann B, Rauschmann M. Effect of lumbar disc replacement on the height of the disc space and the geometry of the facet joints: a cadaver study. J Bone Joint Surg Br. 2010 Apr;92(4):595-601. PubMed PMID: 20357341. Epub 2010/04/02. eng.

20. Takigawa T, Espinoza Orias AA, An HS, Gohgi S, Udayakumar RK, Sugisaki K, et al. Spinal kinematics and facet load transmission after total disc replacement. Spine. 2010 Oct 15;35(22):E1160-6. PubMed PMID: 20881657. Epub 2010/10/01. eng.

21. Shim CS, Lee SH, Shin HD, Kang HS, Choi WC, Jung B, et al. CHARITE versus ProDisc: a comparative study of a minimum 3-year follow-up. Spine. 2007 Apr 20;32(9):1012-8. PubMed PMID: 17450077.

22. Phillips FM, Tzermiadianos MN, Voronov LI, Havey RM, Carandang G, Renner SM, et al. Effect of the Total Facet Arthroplasty System after complete laminectomyfacetectomy on the biomechanics of implanted and adjacent segments. Spine J. 2009 Jan-Feb;9(1):96-102. PubMed PMID: 18440280. Epub 2008/04/29. eng.

23. Palmer DK, Inceoglu S, Cheng WK. Stem fracture after total facet replacement in the lumbar spine: a report of two cases and review of the literature. Spine J. 2011 Jul;11(7):e15-9. PubMed PMID: 21703940. Epub 2011/06/28. eng.

24. Zhu Q, Larson CR, Sjovold SG, Rosler DM, Keynan O, Wilson DR, et al. Biomechanical evaluation of the Total Facet Arthroplasty System: 3-dimensional kinematics. Spine. 2007 Jan 1;32(1):55-62. PubMed PMID: 17202893.

25. Wahnert D, Hoffmeier KL, Lehmann G, Frober R, Hofmann GO, Muckley T. Temperature influence on DXA measurements: bone mineral density acquisition in frozen and thawed human femora. BMC Musculoskelet Disord. 2009;10:25. PubMed PMID: 19239690. Pubmed Central PMCID: 2649884.

26. Patwardhan AG, Havey RM, Meade KP, Lee B, Dunlap B. A follower load increases the load-carrying capacity of the lumbar spine in compression. Spine. 1999 May 15;24(10):1003-9. PubMed PMID: 10332793.

27. Goel VK, Goyal S, Clark C, Nishiyama K, Nye T. Kinematics of the whole lumbar spine. Effect of discectomy. Spine. 1985 Jul-Aug;10(6):543-54. PubMed PMID: 4081869.

28. Gwon JK, Chen J, Lim TH, Han JS, Weinstein JN, Goel VK. In vitro comparative biomechanical analysis of transpedicular screw instrumentations in the lumbar region of the human spine. J Spinal Disord. 1991 Dec;4(4):437-43. PubMed PMID: 1810566. 
29. Wang ST, Goel VK, Fu CY, Kubo S, Choi W, Liu CL, et al. Comparison of two interbody fusion cages for posterior lumbar interbody fusion in a cadaveric model. Int Orthop. 2006 Aug;30(4):299-304. PubMed PMID: 16506025. Pubmed Central PMCID: 2532140.

30. Panjabi MM. Biomechanical evaluation of spinal fixation devices: I. A conceptual framework. Spine. 1988 Oct;13(10):1129-34. PubMed PMID: 3206270.

31. Panjabi MM. Hybrid multidirectional test method to evaluate spinal adjacent-level effects. Clin Biomech (Bristol, Avon). 2007 Mar;22(3):257-65. PubMed PMID: 17196720.

32. Cook DJ, Yeager MS, Cheng BC. Interpedicular travel in the evaluation of spinal implants: an application in posterior dynamic stabilization. Spine. 2012 May 15;37(11):923-31. PubMed PMID: 22024893.

33. Siepe CJ, Zelenkov P, Sauri-Barraza JC, Szeimies U, Grubinger T, Tepass A, et al. The fate of facet joint and adjacent level disc degeneration following total lumbar disc replacement: a prospective clinical, $\mathrm{X}$-ray, and magnetic resonance imaging investigation. Spine. 2010 Oct 15;35(22):1991-2003. PubMed PMID: 20881662. Epub 2010/10/01. eng.

34. Rundell SA, Auerbach JD, Balderston RA, Kurtz SM. Total disc replacement positioning affects facet contact forces and vertebral body strains. Spine. 2008 Nov 1;33(23):2510-7. PubMed PMID: 18978591. Epub 2008/11/04. eng.

35. Demetropoulos CK, Sengupta DK, Knaub MA, Wiater BP, Abjornson C, Truumees $\mathrm{E}$, et al. Biomechanical evaluation of the kinematics of the cadaver lumbar spine following disc replacement with the ProDisc-L prosthesis. Spine. 2010 Jan 1;35(1):26-31. PubMed PMID: 20042953.

36. Dmitriev AE, Gill NW, Kuklo TR, Rosner MK. Effect of multilevel lumbar disc arthroplasty on the operative- and adjacent-level kinematics and intradiscal pressures: an in vitro human cadaveric assessment. Spine J. 2008 Nov-Dec;8(6):918-25. PubMed PMID: 18178528.

37. Kikkawa J, Cunningham BW, Shirado O, Hu N, McAfee PC, Oda H. Biomechanical evaluation of a posterolateral lumbar disc arthroplasty device: an in vitro human cadaveric model. Spine. 2010 Sep 1;35(19):1760-8. PubMed PMID: 20508551.

38. Ingalhalikar AV, Reddy CG, Lim TH, Torner JC, Hitchon PW. Effect of lumbar total disc arthroplasty on the segmental motion and intradiscal pressure at the adjacent level: an in vitro biomechanical study: presented at the 2008 Joint Spine Section Meeting Laboratory investigation. Journal of neurosurgery Spine. 2009 Dec;11(6):715-23. PubMed PMID: 19951025.

39. Sjovold SG, Zhu Q, Bowden A, Larson CR, de Bakker PM, Villarraga ML, et al. Biomechanical evaluation of the Total Facet Arthroplasty System(R) (TFAS(R)): loading as compared to a rigid posterior instrumentation system. Eur Spine J. 2012 Aug;21(8):1660-73. PubMed PMID: 22407270. Pubmed Central PMCID: 3535260.

40. Panjabi MM, Goel VJ. Adjacent-level effects: design of a new test protocol and finite element model simulations of disc replacement. Roundtables in Spine Surg. 2005;1:45-55.

41. Rao RD, David KS, Wang M. Biomechanical changes at adjacent segments following anterior lumbar interbody fusion using tapered cages. Spine. $2005 \mathrm{Dec}$ 15;30(24):2772-6. PubMed PMID: 16371901. Epub 2005/12/24. eng.

42. Martin S, Ghanayem AJ, Tzermiadianos MN, Voronov LI, Havey RM, Renner SM, et al. Kinematics of cervical total disc replacement adjacent to a two-level, straight 
versus lordotic fusion. Spine. 2011 Aug 1;36(17):1359-66. PubMed PMID:

21629170. Epub 2011/06/02. eng.

\section{Corresponding Author}

Brandon G. Santoni, Ph.D., Director of Research, Foundation for Orthopaedic Research \& Education, 13020 N. Telecom Parkway, Tampa, FL 33543

\section{Disclosures}

This study was funded through a research grant from Globus Medical Inc., Audubon, PA). Although institutional support was received, this was an investigator initiated study and the research institution (Foundation for Orthopaedic Research and Education) reserved the rights to all the data in the preparation of this manuscript.

Copyright (C) 2014 ISASS - International Society for the Advancement of Spine Surgery. To see more or order reprints or permissions, see http://ijssurgery.com. 